\section{Remarks on the critical nonlinear high-order heat equation}

Remarks on the high-order heat equation

\author{
Tarek Saanouni
}

Qassim University, Buraidah, Saudi Arabia and

LR03ESO4 Partial Differential Equations and Applications,

Faculty of Science of Tunis, University of Tunis El Manar, Tunis, Tunisia

\begin{abstract}
The initial value problem for a semi-linear high-order heat equation is investigated. In the focusing case, global well-posedness and exponential decay are obtained. In the focusing sign, global and non global existence of solutions are discussed via the potential well method.
\end{abstract}

Keywords Nonlinear high-order heat equation, Global existence, Decay, Blow-up

Paper type Orginal Article

\section{Introduction}

Consider the Cauchy problem for a high-order nonlinear heat equation

$$
\left\{\begin{array}{c}
\dot{u}+(-\Delta)^{k} u+c u=\epsilon|u|^{p-1} u \\
u_{|t=0|}=u_{0}
\end{array}\right.
$$

Higher-order semi-linear and quasilinear diffusion operators occur in applications in thin film theory, non-linear diffusion and lubrication theory, flame and wave propagation, and phase transition at critical Lifschitz points and bistable systems (e.g., the KuramotoSivashinsky equation and the extended Fisher-Kolmogorov equation). See models and references [16].

Here and hereafter $k>1, c \epsilon\{0,1\}, \epsilon= \pm 1, u:=u(t, x)$ is a real-valued function of the variables $(t, x) \in \mathbb{R} \times \mathbb{R}^{n}$ for some integer $n \epsilon\left(2 k, \frac{2 k(1+k)}{k-1}\right)$. The non-linearity satisfies $k \leq p \leq p^{*}:=p_{c}-1:=\frac{n+2 k}{n-2 k}$. The $k$ - Laplacian operator stands for

$$
(-\Delta)^{k}:=(-\Delta)[-\Delta]^{k-1}, \quad(-\Delta)^{0}:=I .
$$

\section{JEL Classification - 35K55}

(C) Tarek Saanouni. Published in the Arab Journal of Mathematical Sciences. Published by Emerald Publishing Limited. This article is published under the Creative Commons Attribution (CC BY 4.0) license. Anyone may reproduce, distribute, translate and create derivative works of this article (for both commercial and non-commercial purposes), subject to full attribution to the original publication and authors. The full terms of this license may be seen at http://creativecommons.org/licences/by/4.0/ legalcode

The publisher wishes to inform readers that the article "Remarks on the critical nonlinear high-order heat equation" was originally published by the previous publisher of the Arab Journal of Mathematical Sciences and the pagination of this article has been subsequently changed. There has been no change to the content of the article. This change was necessary for the journal to transition from the previous publisher to the new one. The publisher sincerely apologises for any inconvenience caused. To access and cite this article, please use Saanouni, T. (2019), "Remarks on the critical nonlinear high-order heat equation”, Arab Journal of Mathematical Sciences, Vol. 26 No. 1/2, pp. 127-152. The original publication date for this paper was 15/03/2019. 
AJMS

$26,1 / 2$

128

The energy space $C\left([0, T], H^{k}\left(\mathbb{R}^{n}\right)\right)$ is naturally adapted to study the high-order heat problem (1.1) using, with a minimal regularity, the following energy identity

$$
\begin{aligned}
\partial_{t} E^{c}(t) & :=\partial_{t} E^{c}(u(t)) \\
& :=\partial_{t}\left[\int_{\mathbb{R}^{n}}\left(\frac{1}{2}\left|\nabla^{k} u(t)\right|^{2}+\frac{c}{2}|u(t)|^{2}-\frac{\epsilon}{1+p}|u(t)|^{1+p}\right) d x\right] \\
& =-\int_{\mathbb{R}^{n}}|\dot{u}(t, x)|^{2} d x
\end{aligned}
$$

If $\epsilon=-1$, the energy is positive and (1.1) is said to be defocusing. For $\epsilon=1$, the energy no longer allows a control of the $H^{k}$ norm of an eventual solution. In such a case, (1.1) is focusing.

In the classical case $k=1$, Eq. (1.1) has been extensively studied in the scale of Lebesgue spaces $L^{q}\left(\mathbb{R}^{n}\right)$. The critical index $q_{c}:=\frac{n(p-1)}{2}$ gives the following three different regimes.

(1) Sub-critical case $q>q_{c} \geq 1$ : Weissler [18] proved local well-posedness in $C([0, T)$; $\left.\left.\left.L^{q}\left(\mathbb{R}^{n}\right)\right) \cap L_{l o c}^{\infty}(] 0, T\right] ; L^{\infty}\left(\mathbb{R}^{n}\right)\right)$. Then Brezis-Cazenave [3] showed unconditional uniqueness.

(2) Critical case $q=q_{c}$ : There are two cases

(a) $q_{c}>p+1$ : local well-posedness holds [3,18];

(b) $q=q_{c}=p+1$ : Weissler [19] proved a conditional well-posedness.

(3) Super-critical case $q<q_{c}$ : There is no solution in any reasonable weak sense $[3,18,19]$. Moreover, uniqueness is lost [10] for the initial data $u_{0}=0$ and for $1+\frac{1}{n}<p<\frac{n+2}{n-2}$.

See [11] for exponential type non-linearity in two space dimensions.

This manuscript seems to be one of few works treating well-posedness issues of the nonlinear high-order heat equation in the energy space $[2,8,9,17]$.

The purpose of this paper is two-fold. First, global well-posedness and exponential decay are established in the defocusing case. Second, in the focusing sign, global and non global existence of solutions are discussed via potential-well method. Comparing with the classical case, we need to operate with various modification due to the highorder Laplacian.

The rest of the paper is organized as follows. Section 2 is devoted to the main results and some tools needed in the sequel. Section 3 deals with local well-posedness of (1.1). Section 4 contains a proof of global existence of solutions in the critical case with small data. Section 5 deals with the associated stationary problem. Section 6 is about global and non global existence of solutions with data in some stable sets in the spirit of Payne and Sattinger [15]. In the last one, the existence of infinitely many non global solutions near the ground state is proved.

We mention that $C$ will be used to denote a constant which may vary from line to line. $A \leqq B$ means that $A \leq C B$ for some absolute constant $C$. For simplicity, denote $\int \cdot d x:=\int_{\mathbb{R}^{n}} \cdot d x, L^{p}:=L^{p}\left(\mathbb{R}^{n}\right)$ is the Lebesgue space endowed with the norm $\|\cdot\|_{p}:=\|\cdot\|_{L^{p}}$ and $\|\cdot\|:=\|\cdot\|_{2}$. The classical Sobolev space is $H^{k, p}:=(I-\Delta)^{\frac{-k}{2}} L^{p}$ and $H^{k}:=H^{k, 2}$ is the energy space. Using Plancherel Theorem, the following norms are equivalent

$$
\|u\|_{H^{k}}:=\left(\int_{\mathbb{R}^{n}}\left(1+|\xi|^{2}\right)^{k}|\widehat{u}(\xi)|^{2} d \xi\right)^{\frac{1}{2}} \simeq\left(\|u\|^{2}+\left\|\nabla^{k} u\right\|^{2}\right)^{\frac{1}{2}}
$$


We denote the real numbers

$$
p_{*}:=1+\frac{4 k}{n}, \quad p^{*}:=p_{c}-1:=\frac{n+2 k}{n-2 k}
$$

Remarks on the high-order heat equation

and we assume here and hereafter that

$$
c=1-\delta_{p}^{p^{*}}=\left\{\begin{array}{lll}
0 & \text { if } & p=p^{*} \\
1 & \text { if } & p \neq p^{*}
\end{array}\right.
$$

Finally, if $T>0$ and $X$ is an abstract functional space, we denote $C_{T}(X):=C([0, T], X)$, $L_{T}^{p}(X):=L^{p}([0, T], X)$ and $X_{r d}$ the set of radial elements in $X$, moreover for an eventual solution to (1.1), we denote $T^{*}>0$ its lifespan.

\section{Background and main results}

In this section we give the main results and some technical tools needed in the sequel.

\subsection{Main results}

Results proved in this paper are listed in what follows.

First, we deal with local well-posedness of the heat problem (1.1) in the energy space.

Theorem 2.1. Take $k>1, n \in\left(2 k, \frac{2 k(1+k)}{k-1}\right), 1<p \leq p^{*}$ and $u_{0} \in H^{k}$. Then, there exist an admissible pair $(q, r)$ in the meaning of Definition 2.8 and a unique maximal solution to (1.1),

$$
u \in L^{q}\left(\left(0, T^{*}\right), H^{k, r}\right)
$$

Moreover,

(1) $u \in C\left(\left[0, T^{*}\right), H^{k}\right)$;

(2) $E(t)=E(0)-\int_{0}^{t} \int_{\mathbb{R}^{n}}|\dot{u}(s, x)|^{2} d x d s$, for any $t \in\left[0, T^{*}\right)$;

(3) if $p<p^{*}$, then

(a) u is unique in $C\left(\left[0, T^{*}\right), H^{k}\right)$;

(b) if $T^{*}<\infty$, then $\lim \sup \|u(t)\|_{H^{k}}=\infty$ and

$$
\|u(t)\|_{H^{k}} \geq \frac{C}{\left(T^{*}-t\right)^{\frac{1}{p-1}-\frac{n-2 k}{4 k}}} ;
$$

(c) if $\epsilon=-1$, then $T^{*}=\infty$ and there exists $\gamma>0$ such that

$$
\|u(t)\|_{H^{k}}=O\left(e^{-\gamma t}\right), \quad \text { when } t \rightarrow \infty .
$$

In the critical case, for small data, there exists a global solution to (1.1).

Theorem 2.2. Take $k>1, n \in\left(2 k, \frac{2 k(1+k)}{k-1}\right)$ and $p=p^{*}$. Then, there exists $\epsilon_{0}>0$ such that if $u_{0} \in \dot{H}^{k}$ satisfies $\left\|u_{0}\right\|_{\dot{H}^{k}} \leq \epsilon_{0}$, the problem (1.1) possesses a unique global solution $u \in C\left(\mathbb{R}_{+}, \dot{H}^{k}\right)$, satisfying the decay 


$$
\lim _{t \rightarrow+\infty}\|u(t)\|_{L^{p}}=0, \quad \text { for all } \quad 2<p<\frac{2 n}{n-2 k} .
$$

Second, we are interested on the focusing case. Using the potential well method due to PayneSattinger [15], we discuss global and non global existence of solutions to (1.1), when the data belongs to some stable sets. Denote the quantities

$$
\bar{\mu}:=\max \{2 \alpha+(n-2 k) \beta, 2 \alpha+n \beta\}, \quad \tilde{\mu}:=\min \{2 \alpha+(n-2 k) \beta, 2 \alpha+n \beta\}
$$

and the set

$$
\mathcal{A}:=\left\{(\alpha, \beta) \in \mathbb{R}_{+}^{*} \times \mathbb{R} \quad \text { s. t } \quad \tilde{\mu}>0 \text { and } \alpha(p-1)+2 k \beta>0\right\} .
$$

The following quantity will be called constraint

$$
K_{\alpha, \beta}^{c}(v)=\frac{1}{2} \int\left[(2 \alpha+(n-2 k) \beta)\left|\nabla^{k} v\right|^{2}+(2 \alpha+n \beta) c|v|^{2}-2\left(\alpha+\frac{n \beta}{1+p}\right)|v|^{1+p}\right] d x .
$$

Take the minimizing problem under constraint

$$
m_{\alpha, \beta}^{c}:=\inf _{0 \neq v \in H_{r d}^{k}}\left\{E^{c}(v), \quad \text { s. t } \quad K_{\alpha, \beta}^{c}(v)=0\right\} .
$$

For easy notation, set

$$
m_{\alpha, \beta}:=m_{\alpha, \beta}^{1}, \quad \in E:=E^{1} \quad \text { and } \quad K_{\alpha, \beta}:=K_{\alpha, \beta}^{1} .
$$

Definition 2.3. We call a ground state to (1.1) any solution to

$$
-(-\Delta)^{k} \phi-c \phi+|\phi|^{p-1} \phi=0, \quad 0 \neq \phi \in H_{r d}^{k}, \quad m_{\alpha, \beta}=E(\phi) .
$$

The existence of ground state is claimed.

Theorem 2.4. Take $k>0, n \geq 2,1<p \leq p^{*}$ and $(\alpha, \beta) \in \mathcal{A}$. So, there exists a ground state solution to (2.2). Moreover, $m^{c}:=m_{\alpha, \beta}^{c}$ is nonzero and independent of $(\alpha, \beta)$.

Denote the spaces

$$
\begin{aligned}
& A_{\alpha, \beta}^{c,+}:=\left\{\phi \in H^{k}, \quad \text { s. t } \quad E^{c}(\phi)<m_{\alpha, \beta}^{c} \quad \text { and } \quad K_{\alpha, \beta}^{c}(\phi) \geq 0\right\} ; \\
& A_{\alpha, \beta}^{c,-}:=\left\{\phi \in H^{k}, \quad \text { s. t } \quad E(\phi)<m_{\alpha, \beta}^{c} \text { and } K_{\alpha, \beta}^{c}(\phi)<0\right\} ; \\
& A_{\alpha, \beta}^{+}:=A_{\alpha, \beta}^{1,+}, \quad A_{\alpha, \beta}^{-}:=A_{\alpha, \beta}^{1,-} .
\end{aligned}
$$

Let us discuss global and non global existence of solutions to the heat problem (1.1).

Theorem 2.5. Take $k>1, n \in\left(2 k, \frac{2 k(1+k)}{k-1}\right), 1<p \leq p^{*}$ and $(\alpha, \beta) \in \mathcal{A}, \epsilon=1$ and $u \in C$ $\left(\left[0, T^{*}\right), H^{k}\right)$ be a maximal solution to (1.1). Then,

(1) if $p<p^{*}$ and $u_{0} \in A_{\alpha, \beta}^{+}$, then $T^{*}=\infty$ and $u(t) \in A_{\alpha, \beta}^{+}$for any time $t \geq 0$. Moreover, for small $\left\|u_{0}\right\|$, there exists $\gamma>0$ such that

$$
\|u(t)\|_{\dot{H}^{k}}=O\left(e^{-\gamma t}\right), \quad \text { when } \quad t \rightarrow \infty ;
$$


(2) if $u_{0} \in A_{\alpha, \beta}^{c,-}$, then u blows-up in finite time.

The last result concerns instability by blow-up for stationary solutions to the heat problem (1.1). Indeed, near ground state, there exist infinitely many data giving non global solutions.

Theorem 2.6. Take $k>1, n \in\left(2 k, \frac{2 k(1+k)}{k-1}\right), \epsilon=1$ and $p_{*}<p \leq p^{*}$. Let $\phi$ be a ground state solution to (2.2). Then, for any $\varepsilon>0$, there exists $u_{0} \in H^{k}$ such that $\left\|u_{0}-\phi\right\|_{H^{k}}<\varepsilon$ and the maximal solution to (1.1) is not global.

2.2 Tools

Let us collect some classical estimates needed forward this manuscript. We start with some technical results about the high-order heat equation. Some useful properties of the free heat kernel are gathered in what follows.

Proposition 2.7. Denoting the free operator associated to the high-order heat equation

$$
T_{k}(t) \phi:=e^{-t(-\Delta)^{k}}:=\mathcal{F}^{-1}\left(e^{-t||^{2 k}}\right) * \phi:=K_{k}(t)^{*} \phi,
$$

yields

(1) $e^{-t(-\Delta)^{k}} u_{0}+\epsilon \int_{0}^{t} e^{-(t-s)(-\Delta)^{k}}|u|^{p-1} u d s$ is the solution to the problem (1.1);

(2) $T_{k} T_{\beta}=T_{k+\beta} \quad T_{k}^{*}=T_{k}$.

Let us recall the so-called Strichartz estimate [20].

Definition 2.8. A couple of real numbers $(q, r)$ is said to be admissible if

$$
q, r \geq 2 \text { and } \frac{2 k}{q}=n\left(\frac{1}{2}-\frac{1}{r}\right) .
$$

Proposition 2.9. Let $n \geq 2, k>0, u_{0} \in L^{2}$ and $(q, r),(q, r)$ two admissible pairs. Then, there exists $C:=C_{q, \tilde{q}}$ such that

$$
\|u\|_{L_{t}^{q}\left(L^{r}\right)} \leq C\left(\left\|u_{0}\right\|+\left\|\dot{u}+(-\Delta)^{k} u\right\|_{L_{t}^{\tilde{q}^{\prime}}}\left(L^{\tilde{r}^{\prime}}\right)\right) .
$$

Proof. Compute

$$
\begin{aligned}
\left(K_{k}(t)\right)(x) & =\mathcal{F}^{-1}\left(e^{-t|\cdot|^{2 k}}\right)(x) \\
& =\frac{1}{t^{2 k}} \mathcal{F}^{-1}\left(e^{-|\cdot|^{2 k}}\right)\left(\frac{x}{t^{\frac{1}{2 k}}}\right) \\
& =\frac{1}{t^{\frac{n}{2 k}}} K\left(\frac{1}{t^{\frac{n}{2 k}}}\right),
\end{aligned}
$$

where $K \in\left(L^{1} \cap L^{\infty}\right)\left(\mathbb{R}^{n}\right)$ (see [7]). Thus,

$$
\left\|T_{k}(t) \phi\right\| \lesssim\|\phi\|, \quad\left\|T_{k}(t) T_{k}^{*}(s) \phi\right\|_{\infty} \lesssim \frac{1}{\mid t-s^{\frac{n}{2 k}}}\|\phi\|_{1} .
$$

The proof is finished via Theorem 1.2 in [12].

Using the above computation via Young inequality, the following smoothing effect yields. 
Lemma 2.10. There exists a positive constant $C$ such that for all $1 \leq r \leq q \leq \infty$, we have

$$
\left\|T_{k}(t) \varphi\right\|_{L^{q}} \leq \frac{C}{t^{\frac{N}{2}\left(\frac{1}{l^{-}}-\frac{1}{q}\right)}}\|\varphi\|_{L^{r}}, \quad \forall t>0, \forall \varphi \varepsilon \in L^{r}\left(\mathbb{R}^{N}\right) .
$$

The following Sobolev injections [1,13] give a meaning to the energy and several computations done in this note.

Lemma 2.11. Let $n \geq 2, k>0$ and $p \in(1, \infty)$. Then,

(1) $W^{k, p}\left(\mathbb{R}^{n}\right) \hookrightarrow L^{q}\left(\mathbb{R}^{n}\right)$ whenever $1<p<q<\infty$, and $\frac{1}{p} \leq \frac{1}{q}+\frac{k}{n}$;

(2) $W^{k}\left(\mathbb{R}^{n}\right) \hookrightarrow L^{q}\left(\mathbb{R}^{n}\right)$ for any $q \in\left[2, \frac{2 n}{n-2 k}\right], n>2 k$

(3) $H_{r d}^{k}\left(\mathbb{R}^{n}\right) \hookrightarrow \hookrightarrow L^{q}\left(\mathbb{R}^{n}\right)$ for any $q \in\left(2, \frac{2 n}{n-2 k}\right), n \geq 2 k$.

The following Gagliardo-Nirenberg inequality is useful throughout the manuscript [14].

Lemma 2.12. Let $n \geq 2, k>0$ and $p, q, r \in(1, \infty)$. Then,

$$
\|\cdot\|_{p} \lesssim\left\|\nabla^{k} \cdot\right\|_{r}^{\theta}\|\cdot\|_{q}^{1-\theta}
$$

for $\frac{1}{p}=\theta\left(\frac{1}{r}-\frac{k}{n}\right)+\frac{1-\theta}{q}$ such that $\theta \in[0,1]$.

In the critical case, recall some properties of the best constant of Sobolev injection $[5,6]$

Proposition 2.13. Take $n \geq 2$ and $0<2 k<n$. Then,

$$
C_{n, k}^{*}:=\inf _{0 \neq u \in \dot{H}^{k}} \frac{\|u\|_{p c}^{2}}{\left\|\nabla^{k} u\right\|^{2}}=\frac{1}{2^{2 k} \pi^{k}} \frac{\Gamma\left(\frac{n}{2}-k\right)}{\Gamma\left(\frac{n}{2}+k\right)} \frac{\Gamma(n)^{\frac{2 k}{n}}}{\Gamma\left(\frac{n}{2}\right)^{\frac{2 k}{n}}} .
$$

Moreover, $u$ is such a minimizer if and only if there exist $c \in \mathbb{R}, \mu>0$ and $x_{0} \in \mathbb{R}^{n}$ such that

$$
u(x)=c\left(\mu^{2}+\left|x-x_{0}\right|^{2}\right)^{-\frac{n-2 k}{2}} .
$$

Let us give an abstract result.

Lemma 2.14. Let $T>0$ and $X \in C\left([0, T], \mathbb{R}_{+}\right)$such that

$$
X \leq a+b X^{\theta} \text { on }[0, T],
$$

where $a b>0, \theta>1, a<\left(1-\frac{1}{\theta}\right)(\theta b)^{\frac{-1}{\theta-1}}$ and $X(0) \leq(\theta b)^{\frac{-1}{\theta-1}}$. Then

$$
X \leq \frac{\theta}{\theta-1} \text { a on }[0, T] \text {. }
$$

Proof. The function $f(x):=b x^{\theta}-x+a$ is decreasing on $\left[0,(b \theta)^{\frac{1}{1-\theta}}\right]$ and increasing on $\left[(b \theta)^{\frac{1}{1-\theta}}, \infty\right)$. The assumptions imply that $f\left((b \theta)^{\frac{1}{1-\theta}}\right)<0$ and $f\left(\frac{\theta}{\theta-1} a\right) \leq 0$. As $f(X(t)) \geq 0$, $f(0)>0$ and $X(0) \leq(b \theta)^{\frac{1}{1-\theta}}$, we conclude the result by a continuity argument.

We close this subsection with a classical result about ordinary differential equations.

Proposition 2.15. Let $\varepsilon>0$. There is no real function $G \in C^{2}\left(\mathbb{R}_{+}\right)$satisfying

$$
G(0)>0, G^{\prime}(0)>0 \text { and } G G^{\prime \prime}-(1+\varepsilon)\left(G^{\prime}\right)^{2} \geq 0 \quad \text { on } \quad \mathbb{R}_{+} \text {. }
$$


Proof. Assume the existence of such a function. Then $\left(G^{-(1+\varepsilon)} G^{\prime}\right)^{\prime} \geq 0$ and

$$
\frac{G^{\prime}}{G^{1+\varepsilon}} \geq \frac{G^{\prime}(0)}{G^{1+\varepsilon}(0)}>0
$$

Integrating on $(0, T)$ the previous inequality, yields

$$
0<\frac{1}{G^{\varepsilon}(T)} \leq \frac{1}{G^{\varepsilon}(0)}-\varepsilon \frac{G^{\prime}(0)}{G^{1+\varepsilon}(0)} T,
$$

which implies that $T<\frac{1}{\varepsilon} \frac{G(0)}{G^{\prime}(0)^{*}}$. This is a contradiction, which achieves the proof.

\section{Local well-posedness}

This section is devoted to proving Theorem 2.1 about local well-posedness of the high-order heat problem (1.1). The result follows by a standard fixed point argument. Take the admissible couple $(q, r):=\left(\frac{4(1+p)}{(p-1)\left(\frac{n}{k}-2\right)}, \frac{p+1}{1+\frac{k}{n}(p-1)}\right)$. Let us start with an intermediary result.

Lemma 3.1. Take $u_{0} \in H^{k}$. There exist $T>0$ and a unique $u \in L_{T}^{q}\left(H^{k, r}\right)$ solution to (1).

Proof. For $R, T>0$ consider the space

$$
X_{T, R}:=\left\{u \in L_{T}^{q}\left(H^{k, r}\right) \quad \text { s. t }\|u\|_{L_{T}^{q}\left(H^{k, r}\right)} \leq R\right\}
$$

endowed with the complete distance

$$
d(u, v):=\|u-v\|_{L_{T}^{q}\left(L^{r}\right)} .
$$

Take the function

$$
\tilde{v}:=\phi(v):=e^{-t(-\Delta)^{k}} u_{0}+\int_{0}^{t} e^{-(t-s)(-\Delta)^{k}}\left(|v|^{p-1} v\right) d s .
$$

We prove that $\phi$ is a contraction of $X_{T, R}$, for some positive $T, R$.

Let $u, v \in X_{T, R}$ and $w:=u-v$. Then, using the equality

$$
\frac{1}{r^{\prime}}=(p-1)\left(\frac{1}{r}-\frac{k}{n}\right)+\frac{1}{r},
$$

we get by Sobolev injection

$$
\begin{aligned}
\left\|w\left(|v|^{p-1}+|u|^{p-1}\right)\right\|_{r^{\prime}} & \leq\|w\|_{r}\left(\|v\|_{\frac{m}{n-k r}}^{p-1}+\|u\|_{\frac{m}{n-k r}}^{p-1}\right) \\
& \leq\|w\|_{r}\left(\|v\|_{H^{k, r}}^{p-1}+\|u\|_{H^{k, r}}^{p-1}\right) .
\end{aligned}
$$

Since $p \leq p^{*}$, there exists $\alpha>0$ such that $\alpha=\infty$ if and only if $p=p^{*}$ and

$$
\frac{1}{\alpha}:=1-\frac{1+p}{q} \text {. }
$$


AJMS

$26,1 / 2$

134
Thanks to Strichartz estimate

$$
\begin{aligned}
\|W\|_{L^{q}\left(I, L^{r}\right)} & \lesssim\left\|w\left(|v|^{p-1}+|u|^{p-1}\right)\right\|_{L q^{\prime}\left(I, L^{r^{\prime}}\right)} \\
& \leq T^{\frac{1}{\alpha}}\|w\|_{L^{q}\left(I, L^{r}\right)}\left[\|v\|_{L^{q}\left(I, L^{r-k}\right)}^{p-1}+\|u\|_{L^{q}\left(I, L^{\left.\frac{m}{r-k}\right)}\right.}^{p-1}\right] \\
& \leq T^{\frac{1}{\alpha}}\|w\|_{L^{q}\left(I, L^{r}\right)}\left[\|v\|_{L^{q}\left(I, H^{k, r}\right)}^{p_{-1}}+\|u\|_{L^{q}\left(I, H^{k, r}\right)}^{p-1}\right] \\
& \leq T^{\frac{1}{\alpha}} R^{p-1}\|w\|_{L^{q}\left(I, L^{r}\right)} .
\end{aligned}
$$

Applying the previous inequality for $v=0$, yields

$$
\begin{aligned}
\|u\|_{L^{q}\left(I, L^{r}\right)} & \leq\left\|e^{-t(-\Delta)^{k}} u_{0}\right\|_{L^{q}\left(I, L^{r}\right)}+T^{\frac{1}{\alpha}} R^{p-1}\|u\|_{L^{q}\left(I, L^{r}\right)} \\
& \leq C\left\|u_{0}\right\|+C T^{\frac{1}{\alpha}} R^{p} .
\end{aligned}
$$

Write now, for $|\alpha|=k$,

$$
\begin{aligned}
\left\|\nabla^{k} \tilde{u}\right\|_{L^{q}\left(I, L^{r}\right)} & \leq\left\|\tilde{u_{0}}\right\|_{\dot{H}^{k}}+\left\|\nabla^{k}\left(u^{p}\right)\right\|_{L^{q^{\prime}}\left(I, L^{r^{\prime}}\right)} \\
& \leq\left\|\tilde{u}_{0}\right\|_{\dot{H}^{k}}+(I)
\end{aligned}
$$

Denoting $P_{j}(\alpha):=\left\{\alpha_{i} \in\left(N^{*}\right)^{j}\right.$ such that $\left.\sum_{i=1}^{j} \alpha_{i}=\alpha\right\}$, we get

$$
(I) \lesssim \sum_{j=1}^{k} \sum_{P_{j}(\alpha)}\left\|u^{p-j} \prod_{i=1}^{j} \partial^{\alpha i} u\right\|_{L q^{\prime}\left(I, L r^{\prime}\right)} .
$$

Take the real numbers

$$
\frac{1}{a_{0}}:=\frac{1}{r}-\frac{k}{n}, \frac{1}{a_{i}}:=\frac{1}{r}-\frac{k-\left|\alpha_{i}\right|}{n}
$$

Then

$$
\frac{p-j}{a_{0}}+\sum_{i=1}^{j} \frac{1}{a_{i}}=\frac{1}{r^{\prime}} .
$$

With Hölder inequality,

$$
\begin{aligned}
(I) & \leq \sum_{j=1}^{k} \sum_{P_{j(\alpha)}}\left\|u^{p-j} \prod_{i=1}^{j} \partial^{\alpha_{i}} u\right\|_{L q^{\prime}\left(I, L r^{\prime}\right)} \\
& \leq T^{\frac{1}{\alpha}} \sum_{j=1}^{k} \sum_{P_{j(\alpha)}}\|u\|_{L q\left(I, L^{a_{0}}\right)}^{p-j} \prod_{i=1}^{j}\left\|\partial^{\alpha_{i}} u\right\|_{L^{q}\left(I, L^{a_{i}}\right)} \\
& \leq T^{\frac{1}{\alpha}} \sum_{j=1}^{k} \sum_{P_{j(\alpha)}}\|u\|_{L q\left(I, L^{n-r k)}\right)}^{p-j} \prod_{i=1}^{j}\|u\|_{L^{q}\left(I, \dot{H}^{\alpha_{i}, a_{i}}\right)^{\cdot}}
\end{aligned}
$$


Taking account of Sobolev embedding

$$
\begin{aligned}
(I) & \leq T^{\frac{1}{\alpha}} \sum_{j=1}^{k} \sum_{P_{j(\alpha)}}\|u\|_{L q\left(I, \dot{H}^{k, r}\right)}^{p-j} \prod_{i=1}^{j}\|u\|_{L q\left(I, \dot{H}^{k, r}\right)} \\
& \leq T^{\frac{1}{\alpha}} \sum_{j=1}^{k} \sum_{P_{j(\alpha)}}\|u\|_{L q\left(I, \dot{H}^{k, r}\right)}^{p-j}\|u\|_{L q\left(I, \dot{H}^{k, r}\right)} \\
& \leq T^{\frac{1}{\alpha}}\|u\|_{L q\left(I, \dot{H}^{k, r}\right)}^{p} \\
& \leq T^{\frac{1}{\alpha}} R^{p} .
\end{aligned}
$$

Remarks on the high-order heat equation

Then

$$
\|\tilde{u}\|_{L^{q}\left(I, H^{k, r}\right)} \leq C\left\|u_{0}\right\|_{H^{k}}+C T^{\frac{1}{\alpha}} R^{p} .
$$

If $p<p^{*}, \frac{1}{\alpha}>0$, so choosing $R:=2 C\left\|u_{0}\right\|_{H^{k}}$ and $T>0$ small enough, it follows that $\phi$ is a contraction of $X_{T, R}$. If $p=p_{c}$ using previous computation with the fact that when $T$ vanishes, $\left\|e^{-t(-\Delta)^{k}} u_{0}\right\|_{L_{T}^{q}\left(H^{k, r}\right)} \rightarrow 0$, it follows that $\phi$ is a contraction of $X_{T, R}$ for small time. Thanks to Picard fixed point theorem, existence of a solution of (1.1) is proved. For uniqueness of such a solution, it is sufficient to apply (3.4) and use a translation argument.

Lemma 3.2. Take $u_{0} \in H^{k}$ and $u \in L_{T}^{q}\left(H^{k, r}\right)$ be a solution of (1.1). Then, $u \in C_{T}\left(H^{k}\right) \cap L_{T}^{q_{1}}$ $\left(H^{k, r_{1}}\right)$ for any admissible couple $\left(q_{1}, r_{1}\right)$.

Proof. Take $0<t_{1}, t_{2}<T$, by Strichartz estimate via the integral formula

$$
\begin{aligned}
\left\|u\left(t_{1}\right)-u\left(t_{2}\right)\right\|_{H^{k}} & \leq\left\|\int_{t_{1}}^{t_{2}} e^{-(t-s)(-\Delta)^{k}}\left(|u|^{p-1} u\right) d s\right\|_{L^{\infty}\left(\left(t_{1}, t_{2}\right), H^{k}\right)} \\
& \leq\left\|u^{p}\right\|_{L q^{\prime}\left(\left(t_{1}, t_{2}\right), H^{k, r^{\prime}}\right)} \\
& \leq\left(t_{1}-t_{2}\right)^{\frac{1}{\alpha}}\|u\|_{L q\left(\left(t_{1}, t_{2}\right), \dot{H}^{k, r}\right)}^{p}
\end{aligned}
$$

This completes the proof.

Let us prove unconditional uniqueness in the sub-critical case. Take $\sigma:=1+p$ and an admissible couple $(a, \sigma)$. With Strichartz estimate

$$
\begin{aligned}
\|\tilde{w}\|_{L^{a}\left(I, L^{\sigma}\right)} & \leq\left\|w\left(|v|^{p-1}+|u|^{p-1}\right)\right\|_{L^{a^{\prime}\left(I, L^{\sigma^{\prime}}\right)}} \\
& \leq T^{1-\frac{2}{a}}\|w\|_{L^{a}\left(I, L^{\sigma}\right)}\left[\|v\|_{L^{\infty}\left(I, L^{\sigma}\right)}^{p-1}+\|u\|_{L^{\infty}\left(I, L^{\sigma}\right)}^{p-1}\right] \\
& \leq T^{1-\frac{2}{a}}\|w\|_{L^{a}\left(I, L^{\sigma}\right)}\left[\|v\|_{L^{\infty}\left(I, H^{k}\right)}^{p-1}+\|u\|_{L^{\infty}\left(I, H^{k}\right)}^{p-1}\right] \\
& \leq T^{1-\frac{2}{a}} R^{p-1}\|w\|_{L^{a}\left(I, L^{\sigma}\right)} .
\end{aligned}
$$


AJMS

$26,1 / 2$

136

The sub-critical condition implies that $\sigma<1+p_{c}$, which gives $a<2$. Then, unconditional uniqueness is established via the last inequality.

Now, for $t \in\left(0, T^{*}\right)$, taking account of (3.5), if there exists $R>0$ such that

$$
C\|u(t)\|_{H^{k}}+C(T-t)^{\frac{1}{\alpha}} R^{p} \leq R,
$$

then, $T<T^{*}$. Thus, for any $R>0$,

$$
C\|u(t)\|_{H^{k}}+C\left(T^{*}-t\right)^{\frac{1}{\alpha}} R^{p} \leq R,
$$

Choosing $R:=2 C\|u(t)\|_{H^{k}}$, it follows that

$$
\left(T^{*}-t\right)^{\frac{1}{\alpha}}\|u(t)\|_{H^{k}}^{p-1} \geq C .
$$

Let us prove that the maximal solution of (1.1) is global in the sub-critical defocusing case. The global existence is a consequence of the energy decay and previous calculations. Let $u \in C\left(\left[0, T^{*}\right), H^{k}\right)$ be the unique maximal solution of (1.1). We prove that $u$ is global. By contradiction, suppose that $T^{*}<\infty$. Consider for $0<s<T^{*}$, the problem

$$
\left(\mathcal{P}_{s}\right)\left\{\begin{array}{l}
\dot{v}+(-\Delta)^{k} v+v+|v|^{p-1} v=0 \\
v(s, .)=u(s, .)
\end{array}\right.
$$

Using the same arguments of local existence, we can find a real $\tau>0$ and a solution $v$ to $\left(\mathcal{P}_{s}\right)$ on $C\left([s, s+\tau], H^{k}\right)$. Thanks to the energy decay, we see that $\tau$ does not depend on $s$. Thus, if we let $s$ be close to $T^{*}$ such that $T^{*}<s+\tau$, this fact contradicts the maximality of $T^{*}$.

Let us prove that $u \in C\left(\mathbb{R}_{+}, H^{k}\right)$, the global solution to (1.1) for $c=-\epsilon=1$ and $1<p<p^{*}$ satisfies an exponential decay in the energy space.

Denoting the quantity $K(u(t)):=\|u(t)\|_{H^{k}}^{2} \int_{\mathbb{R}^{n}}|u(t)|^{1+p} d x$, yields

$$
E(u(t)) \leq K(u(t)) \leq(p+1) E(u(t)) .
$$

On the other hand, for $T>0$,

$$
\begin{aligned}
\int_{t}^{T} K(u(s)) d s & =\frac{1}{2}\left(\|u(t)\|^{2}-\|u(T)\|^{2}\right) \\
& \leq \frac{1}{2}\|u(t)\|^{2} \\
& \leq E(u(t)) .
\end{aligned}
$$

So,

$$
\int_{t}^{T} E(u(s)) d s \lesssim \int_{t}^{T} K(u(s)) d s \lesssim E(u(t)) .
$$

Thus, for some positive real number $T_{0}>0$,

$$
\begin{aligned}
y(t) & :=\int_{t}^{\infty} E(u(s)) d s \\
& \leq E(u(t)) \\
& \leq-T_{0} y^{\prime}(t)
\end{aligned}
$$


This implies that, for $t \geq T_{0}$,

$$
y(t) \leq y\left(T_{0}\right) e^{1-\frac{t}{T_{0}}} \leq T_{0} E\left(u\left(T_{0}\right)\right) e^{1-\frac{t}{T_{0}}} .
$$

Remarks on the high-order heat equation

Taking account of the monotonicity of the energy, for large $T>0$,

$$
\int_{t}^{T} E(u(s)) d s \geq \int_{t}^{t+T_{0}} E(u(s)) d s \geq T_{0} E\left(u\left(t+T_{0}\right)\right) .
$$

Then,

$$
E\left(u\left(t+T_{0}\right)\right) \leq E\left(u\left(T_{0}\right)\right) e^{1-\frac{t}{T_{0}}}
$$

Finally,

$$
\left\|u\left(t+T_{0}\right)\right\|_{H^{k}}^{2} \leq E\left(u\left(t+T_{0}\right)\right) \leq E\left(u\left(T_{0}\right)\right) e^{1-\frac{t}{T_{0}}} .
$$

The proof is finished.

\section{Global well-posedness in the critical case}

This section is devoted to prove Theorem 2.2 about global well-posedness of the critical highorder heat type equation (1.1). Denote the norms

$$
\begin{aligned}
& \|u\|_{Z(I)}:=\|u\|_{L^{2 p^{*}\left(I, L^{2 b^{*}}\right)}} ; \\
& \|u\|_{M(I)}:=\left\|\nabla^{k} u\right\|_{L^{2 p^{*}\left(I, L^{\frac{2 n}{n^{2}+2 k k^{2}}}\right)}} ;
\end{aligned}
$$

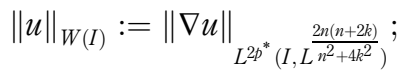

$$
\begin{aligned}
& \|u\|_{N(I)}:=\|\nabla u\|_{L^{2}\left(I, L^{\left.\frac{2 n}{n+2 k}\right)}\right.} .
\end{aligned}
$$

Let us start with an intermediary result.

Lemma 4.1. The following continuous injection holds.

$$
\|u\|_{W(I)} \hookrightarrow\|u\|_{Z(I)} .
$$

Proof. Write

$$
\begin{aligned}
\|u\|_{2 p^{*}} & =\left\|u^{2\left(1-\frac{1}{p_{c}}\right)}\right\|_{P_{c}}^{\frac{p_{c}^{*}}{2 p^{*}}} \\
& \leq\left\|u^{2\left(1-\frac{1}{p_{c}}\right)}\right\|_{\dot{H}^{1}}^{\frac{p_{c}^{*}}{2 \dot{p}^{*}}} \\
& \leq\left\|\nabla u u^{2\left(1-\frac{1}{p_{c}}\right)-1}\right\|^{\frac{p_{c}}{2 p^{*}}} \\
& \leq\left(\left\|u^{2\left(1-\frac{1}{p_{c}}\right)-1}\right\|_{\frac{2 p^{*}}{2\left(1-\frac{1}{p_{c}}\right)-1}}\|\nabla u\|_{\frac{2 p^{*}}{p_{c}-2\left(1-\frac{1}{p_{c}}\right.}}\right)^{\frac{p_{c}}{2 p^{*}}} \\
& \leq\|u\|_{2 p^{*}}^{\frac{p_{c}-2}{2 *^{*}}}\|\nabla u\|_{\frac{\frac{p_{c}}{2 p^{*}}}{p_{c}-2\left(1-\frac{1}{p_{c}}\right)}}
\end{aligned}
$$


Then

$$
\begin{aligned}
& \|u\|_{Z(I)} \lesssim\|\| u\left\|_{2 p^{*}}^{\frac{p c-2}{2 p^{*}}}\right\| \nabla u\left\|_{\frac{2 p^{*}}{p_{c}-2\left(1-\frac{1}{p_{c}}\right)}}^{\frac{p_{c}}{2 p^{*}}}\right\|_{L^{2 p^{*}}(I)} \\
& \begin{array}{l}
\leq u\left\|_{Z(I)}^{\frac{p_{c}-2}{2 p^{*}}}\right\| \nabla u \|^{\frac{p_{c}}{2 p^{*}}} \\
L^{2 p^{*}}\left(I, L^{\frac{2 p^{*}}{p c-2\left(1-\frac{1}{p c}\right)}}\right)
\end{array} \\
& \lesssim\|\nabla u\|_{L^{2 p^{*}}\left(I, L^{\left.\frac{2 p^{*}}{p c-2\left(1-\frac{1}{p c}\right)}\right)}\right.} .
\end{aligned}
$$

Proposition 4.2. Take the critical case $p:=p^{*}$ and I an interval containing zero. There exists $\delta>0$ such that for any $u_{0} \in H^{k}$ satisfying

$$
\left\|e^{-t(-\Delta)^{k}} u_{0}\right\|_{W(I)}<\delta
$$

there exists a unique solution $u \in C\left(I, H^{k}\right)$ to (1.1). Moreover,

$$
\|u\|_{W(I)} \leq 2 \delta, \quad\|u\|_{M(I)}+\|u\|_{L^{\infty}\left(I, H^{k}\right)} \leq C\left(\left\|u_{0}\right\|_{H^{k}}+\delta^{p^{*}}\right) .
$$

Proof. First, we establish the existence of a local solution to (1.1) by a fixed point argument. For $M:=C\left\|u_{0}\right\|_{H^{k}}, T>0$ and $I:=(0, T)$, take the set

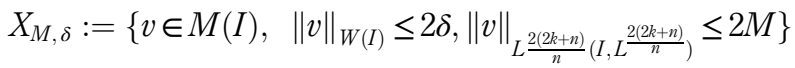

endowed with the complete distance

$$
d(u, v):=\|u-v\|_{L \frac{2(2 k+n)}{n}\left(I, L \frac{2(2 k+n)}{n}\right)} .
$$

Take the function

$$
\tilde{v}:=\phi(v):=e^{-t(-\Delta)^{k}} u_{0}+\int_{0}^{t} e^{-(t-s)(-\Delta)^{k}}|v|^{p_{c}-2} v d s .
$$

Let us prove that for some positive $M, \delta, \phi$ is a contraction of $X_{M, \delta}$.

We establish that $X_{M, \delta}$ is stable by $\phi$ for some small positive $M, \delta$. Let $v \in X_{M, \delta}$. Compute, using Strichartz and Hölder inequalities

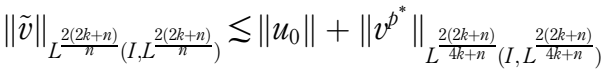

$$
\begin{aligned}
& \lesssim\left\|u_{0}\right\|+\|v\|_{\left.L^{\frac{2(2 k+n)}{n}\left(I, L^{2(2 k+n)}\right.}\right)}\left\|v^{p_{c}-2}\right\|_{L^{\frac{2 k+n}{2 k}\left(I, L^{\frac{2 k+n}{2 k}}\right)}} \\
& \lesssim\left\|u_{0}\right\|+\|v\|_{\left.L^{\frac{2(2 k+n)}{n}(I, L} \frac{2(2 k+n)}{n}\right)}\|v\|_{L^{2 p^{*}}\left(I, L^{2 p^{*}}\right)}^{p_{2}-2} \\
& \leq\left\|u_{0}\right\|+\|v\|_{L^{2(2 k+n)}} \frac{2\left(I, L^{\frac{2(2 k+n)}{n}}\right)}{\| v} \|_{Z(I)}^{p_{c}-2} \\
& \leq M\left(1+\delta^{p_{c}-2}\right)
\end{aligned}
$$


On the other hand

$$
\begin{aligned}
\|\tilde{v}\|_{W(I)} & \leq\left\|e^{-i t(\Delta)^{k}} u_{0}\right\|_{W(I)}+\left\|v|v|^{p_{c}-2}\right\|_{N(I)} \\
& \leq M+\left\|\nabla v v^{p_{c}-2}\right\|_{L^{2}\left(I, \frac{2 n}{L^{2 k+n}}\right)} \\
& \leq M+\|v\|_{Z(I)}^{p_{c}-2}\|v\|_{W(I)} \\
& \leq M+\delta^{p^{*}} .
\end{aligned}
$$

Remarks on the high-order heat equation

Always using Strichartz estimate

$$
\begin{aligned}
\|\tilde{v}\|_{M(I)} & \lesssim\left\|\nabla^{k} u_{0}\right\|+\left\|\nabla^{k}\left(v|v|^{p_{c}-2}\right)\right\|_{L^{2}\left(I, L^{\left.\frac{2 n}{n+2 k}\right)}\right.} \\
& \leq\left\|u_{0}\right\|_{\dot{H}^{k}}+\left\|\nabla^{k}\left(v|v|^{p_{c}-2}\right)\right\|_{L^{2}\left(I, L^{\frac{2 n}{n+2 k}}\right.} .
\end{aligned}
$$

Using Faa-di bruno [4] identities, we get

$$
\nabla^{k}\left(v^{p^{*}}\right)=\sum_{i=1}^{k} v^{p^{*}-i} \sum_{s=1}^{k} \sum_{P_{E}(\nu)} \nu ! \prod_{j=1}^{k} \frac{\left(\partial^{l} j v\right)^{k j}}{k_{j} !\left(l_{j} !\right)^{k j}}
$$

where in $P_{E}(\nu)$, we have $\sum_{j=1}^{k} k_{j}=i, \sum_{j=1}^{k} k_{j} l_{j}=\nu$ and $|\nu|=k$. Then, it is sufficient to estimate the term

$$
\left\|v^{\phi^{*}-i} \prod_{j=1}^{k}\left(\partial^{l} j v\right)^{k j}\right\|_{L^{2}\left(I, L^{\left.\frac{2 n}{n+2 k}\right)}\right.} .
$$

Taking the choice

$$
\alpha_{j}:=\frac{2 p^{*}}{k_{j}}, \quad \frac{1}{\beta_{j}}=k_{j}\left(\frac{\left|l_{j}\right|}{n}+\frac{1}{2 p^{*}}\right)
$$

it follows that

$$
\begin{gathered}
\frac{1}{2}=\frac{p^{*}-i}{2 p^{*}}+\sum_{j=1}^{k} \frac{1}{\alpha_{j}}=\frac{1}{2}-\frac{i}{2 p^{*}}+\sum_{j=1}^{k} \frac{1}{\alpha_{j}} \\
\frac{1}{2}+\frac{k}{n}=\frac{n+2 k}{2 n}=\frac{p^{*}-i}{2 p^{*}}+\sum_{j=1}^{k} \frac{1}{\beta_{j}}=\frac{1}{2}-\frac{i}{2 p^{*}}+\sum_{j=1}^{k} \frac{1}{\beta_{j}} .
\end{gathered}
$$

Thus, with Hölder inequality

$$
\left\|v^{p^{*}-i} \prod_{j=1}^{k}\left(\partial^{l} j v\right)^{k j}\right\|_{L^{2}\left(I, L^{\left.\frac{2 n}{n+2 k}\right)}\right.} \leq\|v\|_{Z(I)}^{p^{*}-i} \prod_{j=1}^{k}\left\|\partial^{l} j v\right\|_{L^{k_{j} \alpha_{j}\left(I, L_{j} \beta_{j}\right)}}^{k j} .
$$

With Sobolev injection, yields

$$
W^{k, \frac{2 n(n+2 k)}{n^{2}+4 k^{2}}} \hookrightarrow W^{k-n\left(\frac{n^{2}+4 k^{2}}{2 n(n+2 k)}-\frac{1}{k_{j} \beta_{j}}\right), k_{j} \beta_{j}} \hookrightarrow W^{\left|l_{j}\right|, k_{j} \beta_{j}} .
$$

This implies that 


$$
\begin{aligned}
\|\tilde{v}\|_{M(I)} & \leq\left\|u_{0}\right\|_{\dot{H}^{k}}+\sum_{i=1}^{k}\|v\|_{Z(I)}^{p^{*}-i} \prod_{j=1}^{k}\left\|\partial^{l} j v\right\|_{\left.L^{k_{j} \alpha_{j}\left(I, L_{j}\right.} k_{j} \beta_{j}\right)}^{k_{j}} \\
& \leq\left\|u_{0}\right\|_{\dot{H}^{k}}+\sum_{i=1}^{k}\|v\|_{Z(I)}^{p^{*}-i}\|v\|_{M(I)}^{i} .
\end{aligned}
$$

This finishes the stability of $X_{M, \delta}$. Now, let $u, v \in X_{M, \delta}$ and $w:=u-v$. Then

$$
\begin{aligned}
& d(u, v) \lesssim\left\|w\left(v^{p_{c}-2}+u^{p_{c}-2}\right)\right\|_{L^{\frac{2(2 k+n)}{4 k+n}\left(I, L^{\frac{2(2 k+n)}{4 k+n}}\right)}} \\
& \leq\|w\|_{L^{\frac{2(2 k+n)}{n}\left(I, L^{\frac{2(2 k+n)}{n}}\right)}}\left[\left\|v^{p_{c}-2}\right\|_{L^{\frac{2 k+n}{2 k}\left(I, L^{\frac{2 k+n}{2 k}}\right)}}+\left\|u^{p_{c}-2}\right\|_{L^{\frac{2 k+n}{2 k}}\left(I, L^{\frac{2 k+n}{2 k}}\right)}\right] \\
& \lesssim\left[\|v\|_{Z(I)}^{p_{c}-2}+\|u\|_{Z(I)}^{p_{c}-2}\right] d(u, v) .
\end{aligned}
$$

Then, using Lemma 4.1, we get

$$
d(u, v) \lesssim \delta^{p_{c}-2} d(u, v) .
$$

This proves the contraction via taking small $\delta, M>0$.

Now, let us prove global existence.

By Strichartz estimate, if $u$ exists on $\left[0, t_{0}\right]$ and satisfies $\left\|u_{0}\right\|_{\dot{H}^{k}}$ small enough, we can use (4.6) to extend $u$ on $\left[t_{0}, t_{0}+1\right]$. Hence, in order to prove global well-posedness, it is sufficient to prove that $\left\|u_{0}\right\|_{\dot{H}^{k}}$ remains small on the whole $\left[0, T^{*}\right)$. Let a positive time $t<T^{*}$. With the decay of energy and Sobolev injection, yields

$$
\begin{aligned}
2 E(u(t)) & =\left\|\nabla^{k} u_{0}\right\|^{2}+\frac{2 \mu}{p_{c}} \int\left|u_{0}\right|^{p_{c}} d x \\
& \lesssim\left\|\nabla^{k} u_{0}\right\|^{2}+\left\|\nabla^{k} u_{0}\right\|^{p_{c}} .
\end{aligned}
$$

Then,

$$
\begin{aligned}
\left\|\nabla^{k} u(t)\right\|^{2} & =2 E(u(t))+\frac{2}{p_{c}} \int|u(t)|^{p_{c}} d x \\
& \lesssim\left\|\nabla^{k} u_{0}\right\|^{2}+\left\|\nabla^{k} u_{0}\right\|^{2 p_{c}}+\left\|\nabla^{k} u(t)\right\|^{p_{c}} .
\end{aligned}
$$

The proof is closed via Lemma 2.14 .

Let us finish this section by proving the decay of solutions. Using the previous proposition, it follows that

$$
u \in M\left(\mathbb{R}_{+}\right) \cap W\left(\mathbb{R}_{+}\right) .
$$

Using previous computation and denoting $v(t):=T_{k}(-t) u(t)$, we get for $t, t^{\prime} \rightarrow+\infty$,

$$
\begin{aligned}
\left\|v(t)-v\left(t^{\prime}\right)\right\|_{\dot{H}^{k}} & \lesssim \int_{t}^{t^{\prime}} T_{k}(-s)\left(|u|^{p c-2} u\right) d s \|_{\dot{H}^{k}} \\
& \leq \sum_{i=1}^{k}\|u\|_{Z\left(t, t^{\prime}\right)}^{p^{*}-i}\|u\|_{M\left(t, t^{\prime}\right)}^{i} \rightarrow 0 .
\end{aligned}
$$

Finally, taking account of Sobolev embeddings and denoting $\phi:=\lim _{t \rightarrow+\infty} v(t)$ in $\dot{H}^{k}$, yields 


$$
\begin{aligned}
\|u(t)\|_{p} & \leq\left\|u(t)-T_{k}(t) \phi\right\|_{p}+\left\|T_{k}(t) \phi\right\|_{p} \\
& \leq\left\|u(t)-T_{k}(t) \phi\right\|_{\dot{H}^{k}}+\left\|T_{k}(t) \phi\right\|_{p} \\
& \leq\|v(t)-\phi\|_{\dot{H}^{k}}+\left\|T_{k}(t) \phi\right\|_{p} .
\end{aligned}
$$

Thanks to the smoothing effect (2.3), the decay is proved.

\section{Existence of a ground state}

The goal of this section is to prove that the elliptic problem

$$
-(-\Delta)^{k} \phi-c \phi+|\phi|^{p-1} \phi=0, \quad \phi \in H_{r d}^{k}
$$

has a ground state in the meaning that it has a nontrivial positive radial solution which minimizes of the energy when $K_{\alpha, \beta}$ vanishes. Let us define the quantities

$$
\begin{gathered}
\phi^{\lambda}:=e^{\alpha \lambda} \phi\left(e^{-\beta \lambda} .\right) ; \\
\mathcal{L}_{\alpha, \beta} E(\phi):=\partial_{\lambda}\left(E\left(\phi^{\lambda}\right)\right)_{\mid \lambda=0}:=K_{\alpha, \beta}(\phi) ; \\
H_{\alpha, \beta}:=\left(1-\frac{\mathcal{L}_{\alpha, \beta}}{\bar{\mu}}\right) E .
\end{gathered}
$$

With a direct calculation

$$
\begin{aligned}
K_{\alpha, \beta}(v) & =\frac{1}{2} \int\left[(2 \alpha+(n-2 k) \beta)\left|\nabla^{k} v\right|^{2}+(2 \alpha+n \beta)|v|^{2}-2\left(\alpha+\frac{n \beta}{1+p}\right)|v|^{1+p}\right] d x \\
& H_{\alpha, \beta}(v)=\frac{1}{2}\left(1-\frac{2 \alpha+(n-2 k) \beta}{\bar{\mu}}\right)\left\|\nabla^{k} v\right\|^{2}+\frac{1}{2}\left(1-\frac{2 \alpha+n \beta}{\bar{\mu}}\right)\|v\|^{2} \\
& +\left[\left(\alpha+\frac{n \beta}{p+1}\right) \frac{1}{\bar{\mu}}-\frac{1}{1+p}\right] \int|v|^{1+p} d x
\end{aligned}
$$

Denote the quadratic part and the nonlinear parts of $K_{\alpha, \beta}$,

$$
K_{\alpha, \beta}^{Q}(v):=\int_{\mathbb{R}^{n}}\left[\left(\alpha+\left(\frac{n}{2}-k\right) \beta\right)\left|\nabla^{k} v\right|^{2}+\left(\alpha+\frac{n}{2} \beta\right)|v|^{2}\right] d x, \quad K^{N}:=K-K^{Q} .
$$

Remark 5.1. Note that,

(1) in this section $(\alpha, \beta) \in \mathcal{A}$;

(2) the proof of Theorem 2.2 is based on several Lemmas;

(3) in this section, we write, for easy notation, $K=K_{\alpha, \beta}, K^{Q}=K_{\alpha, \beta}^{Q}, K^{N}=$ $K_{\alpha, \beta}^{N}, \mathcal{L}=\mathcal{L}_{\alpha, \beta}$ and $H=H_{\alpha, \beta}$.

Lemma 5.2. We have

(1) $m(\mathcal{L} H(\phi), H(\phi))>0$, for all $0 \neq \phi \in H^{k}$;

(2) $\lambda \mapsto H\left(\phi^{\lambda}\right)$ is increasing. 
Proof. Compute

$$
\begin{aligned}
\mathcal{L} H(\phi) & =\mathcal{L}\left(1-\frac{\mathcal{L}}{\bar{\mu}}\right) E(\phi) \\
& =-(\mathcal{L}-\tilde{\mu})(\mathcal{L}-\bar{\mu}) \frac{E(\phi)}{\bar{\mu}}+\tilde{\mu}\left(1-\frac{\mathcal{L}}{\bar{\mu}}\right) E(\phi) \\
& =-(\mathcal{L}-\tilde{\mu})(\mathcal{L}-\bar{\mu}) \frac{E(\phi)}{\bar{\mu}}+\tilde{\mu} H(\phi) .
\end{aligned}
$$

Now, since $(\mathcal{L}-(2 \alpha+\beta(n-2 k)))\left\|\nabla^{k} \phi\right\|^{2}=(\mathcal{L}-(2 \alpha+n \beta))\|\phi\|^{2}=0$, we have $(\mathcal{L}-\tilde{\mu})-$ $(\mathcal{L}-\bar{\mu})\|\phi\|_{H^{k}}^{2}=0$. Moreover $\mathcal{L}\left(|\phi|^{1+p}\right)=(\alpha(1+p)+n \beta)|\phi|^{1+p}$, so because $(\alpha, \beta) \in \mathcal{A}$,

$$
\begin{aligned}
\mathcal{L} H(\phi) & \geq \frac{1}{\bar{\mu}}(L-\tilde{\mu})(L-\bar{\mu}) \int \frac{|\phi|^{1+p}}{1+p} d x \\
& =\frac{\alpha(p-1)(\alpha(p-1)+2 k \beta)}{\bar{\mu}(1+p)} \int|\phi|^{1+p} d x \\
& >0 .
\end{aligned}
$$

The first point of the Lemma follows. The last point is a consequence of the equality $\partial_{\lambda} H\left(\phi^{\lambda}\right)=\mathcal{L} H\left(\phi^{\lambda}\right)$.

The next intermediate result is the following.

Lemma 5.3. Let $\left(\phi_{n}\right)$ be a bounded sequence of $H^{k}-\{0\}$ such that $\lim _{n} K^{Q}\left(\phi_{n}\right)=0$. Then, there exists $n_{0} \in \mathbb{N}$ such that $K\left(\phi_{n}\right)>0$ for all $n \geq n_{0}$.

Proof. Since $(\alpha, \beta) \in \mathcal{A}$, and $K^{Q}\left(\phi_{n}\right)$ vanishes at infinity, by Sobolev injection, we have

$$
K^{N}\left(\phi_{n}\right) \leq\left\|\phi_{n}\right\|_{1+p}^{1+p} \leq\left\|\phi_{n}\right\|_{H^{k}}^{1+p}=o\left(\left\|\phi_{n}\right\|_{H^{k}}^{2}\right) .
$$

Then $K(\phi) \simeq K^{Q}\left(\phi_{n}\right)>0$. The proof is achieved.

The last auxiliary result of this section reads as follows.

\section{Lemma 5.4.}

$$
m_{\alpha, \beta}=\inf _{0 \neq \phi \in H_{r d}^{k}}\{H(\phi), \text { s.t } K(\phi) \leq 0\} .
$$

Proof. Let $m_{1}$ be the right hand side, then it is sufficient to prove that $m \leq m_{1}$. Take $\phi \in H^{k}$ such that $K(\phi)<0$ then by Lemma 5.3, the fact that $\lim _{x \rightarrow-\infty} K^{Q}\left(\phi^{\lambda}\right)=0$ and $\lambda \mapsto H\left(\phi^{\lambda}\right)$ is increasing, there exists $\lambda<0$ such that

$$
K\left(\phi^{\lambda}\right)=0, H\left(\phi^{\lambda}\right) \leq H(\phi) .
$$

The proof is closed.

\section{Proof of Theorem 2.4}

(1) sub-critical case. Let $\left(\phi_{n}\right)$ be a minimizing sequence, namely

$$
0 \neq \phi_{n} \in H_{r d}^{k}, K\left(\phi_{n}\right)=0 \text { and } \lim _{n} H\left(\phi_{n}\right)=\lim _{n} E\left(\phi_{n}\right)=m \text {. }
$$


- First step: $\left(\phi_{n}\right)$ is bounded in $H^{k}$. First case $\beta \geq 0$. Then

$$
\left\|\phi_{n}\right\|_{\dot{H}^{k}}^{2} \lesssim H\left(\phi_{n}\right) \rightarrow m \text {. }
$$

So $\left(\phi_{n}\right)$ is bounded in $\dot{H}^{k}$. Assume that $\lim \sup \left\|\phi_{n}\right\|=\infty$. Then

$$
\begin{aligned}
\left\|\phi_{n}\right\|^{2} & \lesssim K^{Q}\left(\phi_{n}\right) \\
& =-K^{N}\left(\phi_{n}\right) \\
& \leq\left\|\phi_{n}\right\|_{1+p}^{1+p} \\
& \leq\left\|\phi_{n}\right\|^{1+p-\frac{n(p-1)}{2 k}}\left\|\nabla^{k} \phi_{n}\right\|^{\frac{n(p-1)}{2 k}} \\
& \lesssim\left\|\phi_{n}\right\|^{1+p-\frac{n(p-1)}{2 k}} .
\end{aligned}
$$

Remarks on the high-order heat equation

This contradiction achieves this case. Second case $\beta<0$. Using the fact that $\alpha(p-1)+2 k \beta>0$ and $K_{\alpha, \beta}\left(\phi_{n}\right)=0$,

$$
\begin{aligned}
2 \bar{\mu} H\left(\phi_{n}\right) & =-2 k \beta\left\|\phi_{n}\right\|^{2}+\frac{1}{1+p}(\alpha(p-1)+2 k \beta) \int|\phi|^{1+p} d x \\
& \geq \frac{1}{1+p}(\alpha(p-1)+2 k \beta) \int|\phi|^{1+p} d x \\
& \geq\left\|\phi_{n}\right\|_{H^{k}}^{2} .
\end{aligned}
$$

Then, $\left(\phi_{n}\right)$ is bounded in $H^{k}$.

- Second step: $m>0$.

Taking account of the compact injection of the radial Sobolev space $H_{r d}^{k}$ on the Lebesgue space $L^{p}$ for any $2<p<p_{c}$, we take

$$
\phi_{n} \rightarrow \phi \text { in } H^{k} \text { and } \phi_{n} \rightarrow \phi \text { in } L^{p}, \forall p \in\left(2, p_{c}\right) \text {. }
$$

Assume that $\phi=0$, since $\left(\phi_{n}\right)$ is bounded in $H^{k}$, we have

$$
K^{N}\left(\phi_{n}\right) \lesssim\left\|\phi_{n}\right\|_{1+p}^{1+p} \rightarrow 0 .
$$

By Lemma 5.3, $K\left(\phi_{n}\right)>0$ for large $n$ which is absurd. So

$$
\phi \neq 0 \text {. }
$$

With lower semi continuity of $H^{k}$ norm, we have $K(\phi) \leq 0$ and $H(\phi) \leq m$. Using (8), we can assume that $K(\phi)=0$ and $E(\phi)=H(\phi) \leq m$. So that $\phi$ is a minimizer satisfying $0 \neq \phi \in H_{r d}^{k}, K(\phi)=0$ and $E(\phi)=H(\phi)=m$. Thus

$$
m=H(\phi)>0 \text {. }
$$

- $\phi$ is a solution to (2). 
AJMS

Now, there is a Lagrange multiplier $\eta \in \mathbb{R}$ such that $E^{\prime}(\phi)=\eta K^{\prime}(\phi)$. Recall that

$26,1 / 2$ $\mathcal{L}(\phi):=\left(\partial_{\lambda} \phi_{\alpha, \beta}^{\lambda}\right)_{\mid \lambda=0}$ and $\mathcal{L} E(\phi):=\left(\partial_{\lambda} E\left(\phi_{\alpha, \beta}^{\lambda}\right)\right)_{\mid \lambda=0}$. Compute

$$
\begin{aligned}
0=K(\phi) & =\mathcal{L} E(\phi)=\left\langle E^{\prime}(\phi), \mathcal{L}(\phi)\right\rangle \\
& =\eta\left\langle K^{\prime}(\phi), \mathcal{L}(\phi)\right\rangle \\
& =\eta \mathcal{L} K(\phi)=\eta \mathcal{L}^{2} E(\phi) .
\end{aligned}
$$

144

With a previous computation

$$
\begin{aligned}
-(\mathcal{L}-\bar{\mu})(\mathcal{L}-\tilde{\mu}) E(\phi) & =k \frac{p-1}{p+1}(k(p-1)+2 k \beta) \int|\phi|^{1+p} d x \\
& =-\mathcal{L}^{2} E(\phi)-\tilde{\mu} \bar{\mu} E(\phi) \\
& >0 .
\end{aligned}
$$

Thus $\eta=0$ and $E^{\prime}(\phi)=0$. So, $\phi$ is a ground state and $m$ is independent of $\alpha, \beta$.

(2) Critical case. Define the mass less action

$$
\begin{aligned}
K_{\alpha, \beta}^{0}(\phi) & :=\mathcal{L}_{\alpha, \beta} E^{0}(\phi) \\
& =\frac{1}{2}(2 \alpha+(N-2 k) \beta)\left\|\nabla^{k} \phi\right\|^{2}-\left(\alpha+\frac{N \beta}{p_{c}}\right)\|\phi\|_{p_{c}}^{p_{c}} \\
& =\left(\alpha+\frac{N \beta}{p_{c}}\right)\left(\left\|\nabla^{k} \phi\right\|^{2}-\|\phi\|_{p_{c}}^{p_{c}}\right)
\end{aligned}
$$

and the operator

$$
\begin{aligned}
H_{\alpha, \beta}^{0}(\phi) & :=\left(E^{0}-\frac{1}{\alpha p_{c}+N \beta} K_{\alpha, \beta}^{0}\right)(\phi) \\
& =\frac{k}{N}\left\|\nabla^{k} \phi\right\|^{2} .
\end{aligned}
$$

Let $m_{\alpha, \beta}^{0}:=m_{\alpha, \beta}$ for $p=p^{*}$ and the real number

$$
d_{\alpha, \beta}^{0}:=\inf _{0 \neq \phi \in H^{k}}\left\{H_{\alpha, \beta}^{0}(\phi) \text { s. t } K_{\alpha, \beta}^{0}(\phi)<0\right\} .
$$

Claim. $m_{\alpha, \beta}^{0}=d_{\alpha, \beta}^{0}$.

Since $K_{\alpha, \beta}^{0}=0$ implies that $E^{0}=H_{\alpha, \beta}^{0}$, it follows that $m_{\alpha, \beta}^{0} \geq d_{\alpha, \beta}^{0}$. Conversely, take $0 \neq \phi \in H^{k}$ such that $K_{\alpha, \beta}^{0}(\phi)<0$. Thus, when $0<\lambda \rightarrow 0$, we get

$$
\begin{aligned}
K_{\alpha, \beta}^{0}(\lambda \phi) & =\frac{1}{2}(2 \alpha+(N-2 k) \beta) \lambda^{2}\left\|\nabla^{k} \phi\right\|^{2}-\left(\alpha+\frac{N \beta}{p_{c}}\right) \lambda^{p_{c}}\|\phi\|_{p_{c}}^{p_{c}} \\
& \simeq \frac{1}{2}(2 \alpha+(N-2 k) \beta) \lambda^{2}\left\|\nabla^{k} \phi\right\|^{2}>0 .
\end{aligned}
$$


So, there exists $\lambda \in(0,1)$ satisfying $K_{\alpha, \beta}^{0}(\lambda \phi)=0$ and

$$
m_{\alpha, \beta}^{0} \leq H_{\alpha, \beta}^{0}(\lambda \phi)=\lambda^{2} H_{\alpha, \beta}^{0}(\phi) \leq H_{\alpha, \beta}^{0}(\phi) .
$$

Thus, $m_{\alpha, \beta}^{0} \leq d_{\alpha, \beta}^{0}$.

So $m_{\alpha, \beta}^{0}=d_{\alpha, \beta}^{0}$. Because of the definitions of $K_{\alpha, \beta}^{0}$ and $H_{\alpha, \beta}^{0}$, it is clear that $m_{\alpha, \beta}^{0}$ is independent of $(\alpha, \beta)$ and

$$
m:=m_{\alpha, \beta}^{0}=\inf _{0 \neq \phi \in H^{k}}\left\{\frac{k}{N}\left\|\nabla^{k} \phi\right\|^{2} \text { s. t }\left\|\nabla^{k} \phi\right\|^{2}<\|\phi\|_{p_{c}}^{p_{c}}\right\} .
$$

Taking the scaling $\lambda \phi$,

$$
\begin{aligned}
m & =\inf _{0 \neq \phi \in H_{r d}^{k}}\left\{\frac{k}{N} \lambda^{2}\left\|\nabla^{k} \phi\right\|^{2} \text { s. t } \lambda^{2-p_{c}}\left\|\nabla^{k} \phi\right\|^{2}<\|\phi\|_{p_{c}}^{p_{c}}\right\} \\
& =\inf _{0 \neq \phi \in H_{r d}^{k}}\left\{\frac{k}{N}\left\|\nabla^{k} \phi\right\|^{2}\left(\frac{\|\phi\|_{p_{c}}^{p_{c}}}{\left\|\nabla^{k} \phi\right\|^{2}}\right)^{\frac{2}{2-p_{c}}}\right\} \\
& =\frac{k}{N} \inf _{0 \neq \phi \in H_{r d}^{k}}\left\{\left(\frac{\left\|\nabla^{k} \phi\right\|}{\|\phi\|_{p_{c}}}\right)^{\frac{N}{k}}\right\} \\
& =\frac{k}{N}\left(C^{*}\right)^{-\frac{N}{\alpha}} .
\end{aligned}
$$

Here, $C^{*}$ denotes the best constant of the Sobolev injection

$$
\|\phi\|_{p_{c}} \leq C^{*}\left\|\nabla^{k} \phi\right\|
$$

is known [16] to be attained by the following explicit $Q \in \dot{H}^{k}$,

$$
Q(x):=\frac{a}{\left(1+|x|^{2}\right)^{\frac{N}{2}-k}}
$$

which solves the mass less equation

$$
(-\Delta)^{k} Q=Q^{*}
$$

\section{Invariant sets and applications}

This section is devoted to establish Theorem 2.5. The proof is based on two auxiliary results.

Lemma 6.1. The sets $A_{\alpha, \beta}^{c,+}$ and $A_{\alpha, \beta}^{c,-}$ are independent of the couple $(\alpha, \beta)$.

Proof. Take $(\alpha, \beta)$ and $\left(\alpha^{\prime}, \beta^{\prime}\right)$ in $\mathcal{A}$. By Theorem 2.4, the union $A_{\alpha, \beta}^{c,+} \cup A_{\alpha, \beta}^{c,-}$ is independent of $(\alpha, \beta)$. So, it is sufficient to prove that $A_{\alpha, \beta}^{c,+}$ is independent of $(\alpha, \beta)$. If $E^{c}(v)<m$ and $K_{\alpha, \beta}^{c}(v)=0$, then $v=0$. So, $A_{\alpha, \beta}^{c,+}$ is open. The rescaling $v^{\lambda}:=e^{\alpha \lambda} v\left(e^{-\beta \lambda}\right.$.) implies that a neighborhood of zero is in $A_{\alpha, \beta}^{c,+}$. Moreover, this rescaling with $\lambda \rightarrow 0$ gives that $A_{\alpha, \beta}^{c,+}$ is contracted to zero and so it is connected. Now, write 


$$
A_{\alpha, \beta}^{c,+}=A_{\alpha, \beta}^{c,+} \cap\left(A_{\alpha^{\prime}, \beta^{\prime}}^{c,+} \cup A_{\alpha^{\prime}, \beta^{\prime}}^{c,-}\right)=\left(A_{\alpha, \beta}^{c,+}, \cap A_{\alpha^{\prime}, \beta^{\prime}}^{c,+}\right) \cup\left(A_{\alpha, \beta}^{c,+}, \cup A_{\alpha^{\prime}, \beta^{\prime}}^{c,-}\right) .
$$

Since by the definition, $A_{\alpha, \beta}^{c,-}$ is open and $0 \in A_{\alpha, \beta}^{c,+} \cap A_{\alpha^{\prime}, \beta^{\prime}}^{c,+}$, using a connectivity argument, we have $A_{\alpha, \beta}^{c,+}=A_{\alpha^{\prime}, \beta^{\prime}}^{c,+}$. The proof is ended.

Lemma 6.2. The sets $A_{\alpha, \beta}^{c,+}$ and $A_{\alpha, \beta}^{c,-}$ are invariant under the flow of (1.1).

Proof. Take $(\alpha, \beta) \in \mathcal{A}$. Let $u_{0} \in A_{\alpha, \beta}^{c,+}$ and $u \in C_{T^{*}}\left(H^{k}\right)$ be the maximal solution of (1.1). The proof follows with contradiction. Assume that for some time $t_{0} \in\left(0, T^{*}\right), u\left(t_{0}\right) \notin A_{\alpha, \beta}^{c,+}$ and $u(t) \in A_{\alpha, \beta}^{c,+}$ for all $t \in\left(0, t_{0}\right)$. Since the energy is decreasing and $E\left(u\left(t_{0}\right)\right)<m$, then, with a continuity argument, there exists a positive time $t_{1} \in\left(0, t_{0}\right)$ such that $K_{\alpha, \beta}\left(u\left(t_{1}\right)\right)=0$. This contradicts the definition of $m$ and finishes the proof in this case. The proof is similar to $A_{\alpha, \beta}^{c,+}$.

(1) Proof of the first part of Theorem 2.5. Using the two previous Lemmas via a translation argument, we can assume that $u(t) \in A_{1,1}^{+}$for any $t \in\left[0, T^{*}\right)$. Taking account of the definition of $m$, we get

$$
\begin{aligned}
& m>E(u(t)) \\
& >E(u(t))-\frac{1}{2+n} K_{1,1}(u(t)) \\
& =\frac{\alpha}{2+n}\left\|\nabla^{k} u(t)\right\|^{2}+\frac{p-1}{(1+p)(2+n)}\|u(t)\|_{1+p}^{1+p} .
\end{aligned}
$$

This implies, via decay of the equality

$$
\partial_{t}\left(\|u(t)\|^{2}\right)=2 K_{1,0}(u(t))<0,
$$

that

$$
\sup _{\left[0, T^{*}\right]}\|u(t)\|_{H^{k}}<\infty
$$

Then, $u$ is global.

Now, we prove an exponential decay. For small $\left\|u_{0}\right\|$, since $\sup _{t}\|u(t)\|_{H^{k}} \leq 1$, we get using Gagliardo-Nirenberg inequality in Lemma 2.12,

$$
\begin{aligned}
K_{1,0}(u(t)) & =\|u(t)\|_{H^{k}}^{2}-\int_{\mathbb{R}^{n}}|u(t)|^{1+p} d x \\
& \geq\|u(t)\|^{2}+\|u(t)\|_{\dot{H}^{k}}^{2}-C\|u(t)\|^{p+1-\frac{n(p-1)}{2 k}}\|u(t)\|_{\dot{H}^{k}}^{\frac{n(p-1)}{2 k}} \\
& \geq\|u(t)\|^{2}+\|u(t)\|_{\dot{H}^{k}}^{2}\left(1-C\left\|u_{0}\right\|^{p+1-\frac{n(p-1)}{2 k}}\|u(t)\|_{\dot{H}^{k}}^{\frac{n(p-1)}{2 k}}\right) \\
& \geq C\|u(t)\|_{\dot{H}^{k}}^{2} \\
& \geq C E(u(t)) .
\end{aligned}
$$


On the other hand

$$
\begin{aligned}
E(u(t)) & =\frac{1}{2}\|u(t)\|_{H^{k}}^{2}-\frac{1}{1+p} \int_{\mathbb{R}^{n}}|u(t)|^{1+p} d x \\
& =\frac{1}{2}\|u(t)\|_{H^{k}}^{2}-\frac{1}{1+p}\left(\|u(t)\|_{H^{k}}^{2}-K_{1,0}(u(t))\right) \\
& =\left(\frac{1}{2}-\frac{1}{1+p}\right)\|u(t)\|_{H^{k}}^{2}+\frac{1}{1+p} K_{1,0}(u(t)) \\
& \geq C \max \left\{K_{1,0}(u(t)),\|u(t)\|_{H^{k}}^{2}\right\} .
\end{aligned}
$$

Moreover, for $T>0$,

$$
\begin{aligned}
\int_{t}^{T} K_{1,0}(u(s)) & d s \\
& =\frac{1}{2}\left(\|u(t)\|^{2}-\|u(T)\|^{2}\right) \\
& \leq \frac{1}{2}\|u(t)\|^{2} \\
\leq & C E(u(t)) .
\end{aligned}
$$

So,

$$
\int_{t}^{T} E(u(s)) d s \lesssim \int_{t}^{T} K_{1,0}(u(s)) d s \lesssim E(u(t)) .
$$

Thus, for some positive real number $T_{0}>0$,

$$
\begin{aligned}
y(t): & =\int_{t}^{\infty} E(u(s)) d s \\
& \leq E(u(t)) \\
& \leq-T_{0} y^{\prime}(t)
\end{aligned}
$$

This implies that, for $t \geq T_{0}$,

$$
y(t) \leq y\left(T_{0}\right) e^{1-\frac{t}{T_{0}}} \leq T_{0} E\left(u\left(T_{0}\right)\right) e^{1-\frac{t}{T_{0}}} .
$$

Taking account of the monotonicity of the energy, for large $T>0$,

$$
\int_{t}^{T} E(u(s)) d s \geq \int_{t}^{t+T_{0}} E(u(s)) d s \geq T_{0} E\left(u\left(t+T_{0}\right)\right) .
$$

Then,

$$
E\left(u\left(t+T_{0}\right)\right) \leq E\left(u\left(T_{0}\right)\right) e^{1-\frac{t}{T_{0}}} .
$$

Finally,

$$
\left\|u\left(t+T_{0}\right)\right\|_{H^{k}}^{2} \leq E\left(u\left(t+T_{0}\right)\right) \leq E\left(u\left(T_{0}\right)\right) e^{1-\frac{t}{T_{0}}} .
$$

The proof is finished. 
AJMS

$26,1 / 2$

(2) Proof of the second part of Theorem 2.4. Using the two previous Lemmas via a translation argument, we can assume that $u(t) \in A_{1, \lambda}^{c,-}$ for any $t \in\left[0, T^{*}\right)$ and any $\lambda>0$. Take the real function

$$
L(t):=\frac{1}{2} \int_{0}^{t}\|u(s)\|^{2} d s, \quad t \in\left[0, T^{*}\right) .
$$

\section{8}

Using Eq. (1.1), a direct computation gives

$$
L^{\prime \prime}(t)=\int_{\mathbb{R}^{n}} \dot{u} u d x=-\|u(t)\|_{\dot{H}^{k}}^{2}-c\|u(t)\|^{2}+\int_{\mathbb{R}^{n}}|u|^{1+p} d x .
$$

We discuss two cases.

(a) First case: $E^{c}\left(u_{0}\right)>0$. For any $\lambda>0$,

$$
H_{1, \lambda}(u)=\frac{1}{2+N \lambda}\left[k \lambda\left\|\nabla^{k} u\right\|^{2}+\frac{p-1}{p+1} \int_{\mathbb{R}^{n}}|u|^{p+1} d x\right]>m .
$$

Thus, for any $\varepsilon>0$,

$$
\begin{aligned}
L^{\prime \prime} & =\varepsilon\left\|\nabla^{k} u\right\|^{2}-(1+\varepsilon)\left\|\nabla^{k} u\right\|^{2}-c\|u(t)\|^{2}+\int_{\mathbb{R}^{n}}|u|^{p+1} d x \\
& >\frac{\varepsilon}{k}\left[\left(\frac{2}{\lambda}+n\right) m-\frac{1}{\lambda} \frac{p-1}{p+1} \int_{\mathbb{R}^{n}}|u|^{p+1} d x\right] \\
& -2(1+\varepsilon)\left[E^{c}\left(u_{0}\right)+\frac{1}{2(1+p)} \int|u|^{p+1} d x\right] \\
& +2(1+\varepsilon) \int_{0}^{t}\|\dot{u}(s)\|^{2} d s+\int_{\mathbb{R}^{n}}|u|^{p+1} d x \\
> & {\left[\frac{\varepsilon}{k}\left(\frac{2}{\lambda}+n\right) m-2(1+\varepsilon) E^{c}\left(u_{0}\right)\right]+\left(1-\frac{1+\varepsilon}{1+p}-\frac{\varepsilon(p-1)}{k \lambda(p+1)}\right) } \\
& \times \int_{\mathbb{R}^{n}}|u|^{p+1} d x+2(1+\varepsilon) \int_{0}^{t}\|\dot{u}(s)\|^{2} d s \\
& :=(I)+\frac{(I I)}{p+1} \int_{\mathbb{R}^{n}}|u|^{p+1} d x+2(1+\varepsilon) \int_{0}^{t}\|\dot{u}(s)\|^{2} d s .
\end{aligned}
$$

Taking $\lambda:=a \varepsilon$ and $\gamma:=m-E^{c}\left(u_{0}\right)$, we get

$$
\begin{aligned}
(I) & =2 \gamma(1+\varepsilon)+m\left[\frac{2}{k a}-2+\varepsilon\left(-2+\frac{N}{k}\right)\right] \\
& =\varepsilon\left(2 \gamma-2 m+\frac{N m}{k}\right)+2 m\left(\frac{1}{k a}-1\right)+2 \gamma .
\end{aligned}
$$


On the other hand,

$$
\begin{aligned}
(I I) & =p+1-(1+\varepsilon)-\frac{p-1}{k a} \\
& =(p-1)\left(1-\frac{1}{k a}\right)+1-\varepsilon .
\end{aligned}
$$

The choice $\frac{1}{k} \frac{p-1}{p-\varepsilon}<a<\frac{1}{k}$, via $\varepsilon>0$ near to zero implies that the terms $(I)$ and $(I I)$ are non negative. Thus,

$$
L^{\prime \prime}>2(1+\varepsilon) \int_{0}^{t}\|u \dot{u}(s)\|^{2} d s .
$$

Thanks to Cauchy-Schwarz inequality, it follows that

$$
\begin{aligned}
L L^{\prime \prime} & >(1+\varepsilon)\|\dot{u}\|_{L_{t}^{2}\left(L^{2}\right)}^{2}\|u\|_{L_{t}^{2}\left(L^{2}\right)}^{2} \\
& >(1+\varepsilon)\|u \dot{u}\|_{L_{t}^{1}\left(L^{1}\right)}^{2} \\
& >(1+\varepsilon) L^{\prime 2} .
\end{aligned}
$$

Indeed, if $L(t)=0$ for some positive time, we get $u_{0}=E\left(u_{0}\right)=0$, which is a contradiction. Thus

$$
\left.\left(L^{-\varepsilon}\right)^{\prime \prime}=-\varepsilon L^{-\varepsilon-2}\left[L^{\prime \prime} L-(1+\varepsilon)\left(L^{\prime \prime}\right)^{2}\right]\right\rangle 0 .
$$

Taking account of Proposition 2.15, for some finite time $T>0$,

$$
\limsup _{t \rightarrow T} \int_{0}^{T}\|u(s)\|^{2} d s=\infty .
$$

Thus, $T^{*}<\infty$ and $u$ is not global. This ends the proof.

(b) Second case: $E^{c}\left(u_{0}\right) \leq 0$. Compute

$$
\begin{aligned}
L^{\prime \prime} & =-\|u\|_{\dot{H}^{k}}^{2}-c\|u\|^{2}+\int_{\mathbb{R}^{n}}|u|^{p+1} d x \\
& \geq(2+\varepsilon)\left(\int_{\mathbb{R}^{n}} \frac{|u|^{p+1}}{p+1} d x-\frac{1}{2}\|u\|_{\dot{H}^{k}}^{2} \frac{c}{2}\|u\|^{2}\right) \\
& \geq-(2+\varepsilon) E^{c}(u) .
\end{aligned}
$$

So, thanks to the identity $\dot{E}^{c}(u)=-\|\dot{u}\|^{2}$, we get

$$
L^{\prime \prime} \geq(2+\varepsilon)\left(\|\dot{u}\|_{L_{t}^{2}\left(L^{2}\right)}^{2}-E^{c}\left(u_{0}\right)\right) .
$$

Now, the proof goes by contradiction assuming that $T^{*}=\infty$.

Claim 1. There exists $t_{1}>0$ such that $\int_{0}^{t_{1}}\|\dot{u}(s)\|^{2} d s>0$.

Indeed, otherwise $u(t)=u_{0}$ almost everywhere and solves the elliptic stationary equation $(-\Delta)^{k} u+c u=|u|^{p-1} u$. Therefore, $\|u\|_{\dot{H}^{k}}^{2}+C\|u\|^{2}=\int_{\mathbb{R}^{n}}|u|^{p+1} d x$ and 


$$
\left\|u_{0}\right\|_{\dot{H}^{k}}^{2}+c\left\|u_{0}\right\|^{2}-\frac{2}{p+1} \int_{\mathbb{R}^{n}}\left|u_{0}\right|^{p+1} d x=\left(1-\frac{2}{p+1}\right) \int_{\mathbb{R}^{n}}\left|u_{0}\right|^{p+1} d x=2 E\left(u_{0}\right) \leq 0 .
$$

Then, $u_{0}=0$ which contradicts the fact that $K_{0,1}\left(u_{0}\right)<0$.

Claim 2. For any $0<\alpha<1$, there exists $t_{\alpha}>0$ such that

$$
\left(L^{\prime}-L^{\prime}(0)\right)^{2} \geq \alpha L^{\prime 2}, \quad \text { on }\left(t_{\alpha}, \infty\right) .
$$

The claim immediately follows from the first one and (6.10) observing that

$$
\lim _{t \rightarrow \infty} L(t)=\lim _{t \rightarrow \infty} L^{\prime}(t)=+\infty .
$$

Claim 3. One can choose $\alpha=\alpha(\varepsilon)$ such that

$$
L L^{\prime \prime} \geq(1+\alpha) L^{\prime 2}, \quad \text { on }\left(t_{\alpha}, \infty\right) \text {. }
$$

Indeed, we have

$$
\begin{aligned}
L L^{\prime \prime} & \geq \frac{2+\varepsilon}{2}\|u\|_{L_{t}^{2}\left(L^{2}\right)}^{2}\|\dot{u}\|_{L_{t}^{2}\left(L^{2}\right)}^{2} \\
& \geq \frac{2+\varepsilon}{2}\|u \dot{u}\|_{L_{t}^{1}\left(L^{1}\right)}^{2} \\
& \geq \frac{2+\varepsilon}{2}\left(L^{\prime}-L^{\prime}(0)\right)^{2} \\
& \geq \frac{(2+\varepsilon) \alpha}{2} L^{\prime 2},
\end{aligned}
$$

where we used (6.10) in the first estimate, Cauchy-Schwarz inequality in the second and Claim 2 in the last one. Now choosing $\alpha$ such that $1<\frac{(2+\varepsilon) \alpha}{2}:=1+\varepsilon$, we get

$$
L L^{\prime \prime}>(1+\varepsilon) L^{\prime 2}, \text { for large time. }
$$

Thanks to Proposition 2.15, this ordinary differential inequality blows up in finite time and contradicts our assumption that the solution is global. This ends the proof.

\section{Strong instability}

This section is devoted to prove Theorem 2.5 about strong instability of stationary solutions to (1.1). Take here and hereafter $c=\epsilon=1$. Denote the scaling $u_{\lambda}:=\lambda^{\frac{N}{2}} u(\lambda$.). Let us write an auxiliary result.

Lemma 7.1. Let $u \in H^{k}$ such that $K_{1,-\frac{2}{n}}(u) \leq 0$. Then, there exists $\lambda_{0} \leq 1$ such that

(1) $K_{1,-\frac{2}{n}}\left(u_{\lambda_{0}}\right)=0$;

(2) $\lambda_{0}=1$ if and only if $K_{1,-\frac{2}{n}}(u)=0$;

(3) $\frac{\partial}{\partial \lambda} E\left(u_{\lambda}\right)>0$ for $\lambda \in\left(0, \lambda_{0}\right)$ and $\frac{\partial}{\partial \lambda} E\left(u_{\lambda}\right)<0$ for $\lambda \in\left(\lambda_{0}, \infty\right)$;

(4) $\lambda \rightarrow E\left(u_{\lambda}\right)$ is concave on $\left(\lambda_{0}, \infty\right)$;

(5) $\frac{\partial}{\partial \lambda} E\left(u_{\lambda}\right)=\frac{N}{2 \lambda} K_{1,-\frac{2}{n}}\left(u_{\lambda}\right)$. 
Proof. With direct computations, we have

$$
\begin{gathered}
K_{1,-\frac{2}{n}}\left(u_{\lambda}\right)=\frac{2 k \lambda^{2 k}}{N}\left\|\nabla^{k} u\right\|^{2}-\left(1-\frac{2}{1+p}\right) \lambda^{\frac{N}{2}(p-1)} \int_{\mathbb{R}^{n}}|u|^{1+p} d x ; \\
\partial_{\lambda} E\left(u_{\lambda}\right)=\frac{N}{2 \lambda} K_{1,-\frac{2}{n}}\left(u_{\lambda}\right),
\end{gathered}
$$

which proves (5). Now

$$
K_{1,-\frac{2}{n}}\left(u_{\lambda}\right)=\frac{2 k \lambda^{2 k}}{N}\left[\left\|\nabla^{k} u\right\|^{2}-\frac{N}{k}\left(\frac{1}{2}-\frac{1}{1+p}\right) \lambda^{\frac{N}{2}(p-1)-2 k} \int_{\mathbb{R}^{n}}|u|^{1+p} d x\right] .
$$

A monotonicity argument via the inequality $p<p^{*}$ closes the proof of (1), (2) and (3). For (4), it is sufficient to compute using (3).

Lemma 7.2. Let $\phi$ be a ground state solution of (2.2), $\lambda>1$ a real number close to one and $u_{\lambda} \in C\left(\left[0, T^{*}\right), H^{k}\right)$ be the solution to (1.1) with data $\phi_{\lambda}$. Then, for any $t \in\left(0, T^{*}\right)$,

$$
E\left(u_{\lambda}(t)\right)<E(\phi) \text { and } K_{1,-\frac{2}{n}}\left(u_{\lambda}(t)\right)<0 \text {. }
$$

Proof. By Lemma 7.1, we have

$$
E\left(\phi_{\lambda}\right)<E(\phi) \text { and } K_{1,-\frac{2}{n}}\left(\phi_{\lambda}\right)<0 .
$$

Moreover, thanks to the decay of energy, it follows that for any $t>0$,

$$
E\left(u_{\lambda}(t)\right) \leq E\left(\phi_{\lambda}(t)\right)<E(\phi) .
$$

Then $K_{1,-\frac{2}{n}}\left(u_{\lambda}(t)\right) \neq 0$ because $\phi$ is a ground state. Finally $K_{1,-\frac{2}{n}}\left(u_{\lambda}(t)\right)<0$ with a continuity argument.

Now, we are ready to prove the instability result.

Take $u_{\lambda} \in C_{T^{*}}\left(H^{k}\right)$ the maximal solution to (1.1) with data $\phi_{\lambda}$, where $\lambda>1$ is close to one and $\phi$ is a ground state solution to (2.2). With the previous Lemma, we get

$$
u_{\lambda}(t) \in A_{1,-\frac{2}{n}}^{-} \text {, for any } t \in\left(0, T^{*}\right) .
$$

Then, using Theorem 2.5, it follows that

The proof is finished via the fact that

$$
\lim _{t \rightarrow T^{*}} \sup \left\|u_{\lambda}(t)\right\|_{H}^{k}=\infty .
$$

$$
\lim _{\lambda \rightarrow 1}\left\|\phi_{\lambda}-\phi\right\|_{H^{k}}=0
$$

\section{References}

[1] D.R. Adams, Sobolev Spaces, Academic Press, New York, 1975.

[2] F. Bernis, A. Friedman, Higher order nonlinear degenerate parabolic equations, J. Differential Equations 83 (1990) 179-206.

[3] H. Brezis, T. Cazenave, A nonlinear heat equation with singular initial data, J. d'Anal. Math. 68 (1996) 73-90.

[4] G.M. Constantine, T.H. Savitis, A multivariate Faa Di Bruno formula with applications, Trans. Amer. Math. Soc. 348 (2) (1996) 503-520. 
AJMS

$26,1 / 2$

152
[5] A. Cotsiolis, N.K. Tavoularis, Best constants for Sobolev inequalities for higher order fractional derivatives, J. Math. Anal. Appl. 295 (2004) 225-236.

[6] J. Davila, M.D. Pino, Y. Sire, Non degeneracy of the bubble in the critical case for non local equations, Proc. Amer. Math. Soc. 141 (2013) 3865-3870.

[7] S.D. Eidel'man, Parabolic systems, in: Translated from the Russian by Scripta Tech- nica, NorthHolland Publishing, London, Amsterdam, 1969.

[8] V.A. Galaktionov, Critical global asymptotics in high-order semilinear parabolic equations, Int. J. Math. Math. Sci. 60 (2003) 3809-3825.

[9] V.A. Galaktionov, S.I. Pohozaev, Existence and blow-up for higher-order semi- linear parabolic equations: Majorizing order-preserving operators, Indiana Univ. Math. J. 51 (6) (2002) 1321-1338.

[10] A. Haraux, F.B. Weissler, Non uniqueness for a semilinear initial value problem, Indiana Univ. Math. J. 31 (1982) 167-189.

[11] S. Ibrahim, M. Majdoub, R. Jrad, T. Saanouni, Local well posedness of a $2 D$ semilinear heat equation, Bull. Belg. Math. Soc. Simon Stevin 21 (3) (2014) 535-551.

[12] M. Keel, T. Tao, Endpoint Strichartz estimates, Amer. J. Math. 120 (1998) 955-980.

[13] P.L. Lions, Symetrie et compacité dans les espaces de Sobolev, J. Funct. Anal. 49 (1982) 315-334.

[14] L. Nirenberg, On elliptic partial differential equations, Ann. Sc. Norm. Super Pisa Cl. Sci. 13 (1955) $116-162$.

[15] L.E. Payne, D.H. Sattinger, Saddle points and instability of nonlinear hyperbolic equations, Israel J. Math. 22 (3-4) (1975) 273-303.

[16] L.A. Peletier, W.C. Troy, Spatial Patterns. Higher Order Models in Physics and Mechanics, in: Progress in Nonlinear Differential Equations and their Appli- cations, vol. 45, Birkhuser Boston, Massachusetts, 2001.

[17] T. Saanouni, Global well-posedness and finite time blow-up of some heat type equations, Proc. Edinb. Math. Soc. 60 (2017) 481-497.

[18] F.B. Weissler, Local existence and nonexistence for a semilinear parabolic equation in $L^{p}$, Indiana Univ. Math. J. 29 (1980) 79-102.

[19] F.B. Weissler, Existence and nonexistence of global solutions for a semilinear heat equation, Israel J. Math. 38 (1981) 29-40.

[20] Z. Zhai, Strichartz type estimates for fractional heat equations, J. Math. Anal. Appl. 356 (2009) $642-658$.

\section{Corresponding author}

Tarek Saanouni can be contacted at: Tarek.saanouni@ipeiem.rnu.tn

For instructions on how to order reprints of this article, please visit our website:

www.emeraldgrouppublishing.com/licensing/reprints.htm

Or contact us for further details: permissions@emeraldinsight.com 